\title{
Hydration Effect on the Intrinsic Magnetism of Natural Deoxyribonucleic Acid as Studied by EMR Spectroscopy and SQUID Measurements
}

\author{
Young-Wan Kwon, Chang Hoon Lee, Eui-Doo Do, Dong Hoon Choi, Jung-Il Jin, Jun Sung Kang, ${ }^{\ddagger}$ and Eui-Kwan Koh ${ }^{\ddagger}$ \\ Department of Chemistry, Korea Lniversity, Seoul 136-701, Korea. "E-mail: jimakorea.ac.kr \\ "Department of Polymer Science and Engineering, Chosin Lniversity, Gwang-Ju 501-759, Korea \\ ¥Korea Basic Science Institute-Seoul Branch, Korea Lniversitv, Seoul 136-701, Korea \\ Received Mav $\mathrm{H}_{1} 2008$
}

\begin{abstract}
The hydration effect on the intrinsic magnetism of natural salmon double-strand DNA was explored using electron magnetic resonance (EMR) spectroscopy and superconducting quantum interference device (SQUD) magnetic measurements. We learned from this study that the magnetic properties of DNA are roughly classified into two distinct groups depending on their water content: One group is of higher water content in the range of 2.6-24 water molecules per nucleotide (wpn), where all the EMR parameters and SQUID susceptibilities are dominated by spin species experiencing quasi one-dimensional diffusive motion and are independent of the water content. The other group is of lower water content in the range of 1.4-0.5 wpn. In this group. the magnetic properties are most probably dominated by cyclotron motion of spin species along the helical $\pi$-way, which is possible when the momentum scattering time $\left(\tau_{k}\right)$ is long enough not only to satisfy the cyclotron resonance condition $\left(\omega_{i} \tau_{k}>1\right)$ but also to induce a constnictive interference between the neighboring double helices. The same effect is reflected in the S-shaped magnetization-magnetic field strength $(\mathrm{M}-\mathrm{H})$ curves superimposed with the linear background obtained by SQUD measurements. which leads to larger susceptibilities at $1000 \mathrm{G}$ when compared with the values at $10.000 \mathrm{G}$. In particular. we propose that the spin-orbital coupling and Faraday's mutual inductive effect can be utilized to interpret the dimensional crossover of spin motions from quasi ID in the hydrate state to $3 \mathrm{D}$ in the dry state of dsDNA.
\end{abstract}

Key Words : Hydration effect. Intrinsic magnetism. Natural DNA, Electron magnetic resonance. SQUID

\section{Introduction}

Traditionally. deosyribonucleic acid (DNA) is regarded as the treasury of hereditary information and has been the center of contemporary biological science and technologies. On the other hand scientific interest in the physico-chemical properties of DNA and modified DNA as a class of naturally occurring polymers is growing rapidly. Especially its functional properties such as charge transport ${ }^{1-4}$ optical properties ${ }^{5-8}$ and magnetic properties ${ }^{9-14}$ are attracting a growing interest closely related to nanoscience and nanoteclunology. Use of DNA as templates ${ }^{15-19}$ in the preparation of metallic nano particles and nanowires is another important advance in this area of research. Most of the specific and often unexpected observations made on the properties of natural and modified DNA can be ascribed to their unique structural features: double stranded helical structures and the stacking planes of the constitutive base pairs.

In the late 1950's and early 1960's there were several reports that describe EMR signals in unirradiated DNA samples. Bliumenfeld and coworkers ${ }^{9}$ were the first to report the EMR signals for unirradiated DNA. Earlier the EMR signals for irradiated DNA were described by Shields and Gordy. ${ }^{21}$ as well as Sheng and coworkers. ${ }^{21}$ Müller and coworkers $:$ also observed the appearance of a broad EMR spectrum in highly purified phage $E$. coli $\mathrm{T} 1$. The origins of the peaks were the subject of heated discussions and controversies among the scientists at that time. There were two schools with different opinions: one believed that the EMR signals were from magnetic impurities such as iron compounds. and the others insisted that the signals are from the intrinsic structural characteristics of the DNA. The controversial views. however. were not clearly resolved and the discussion on the origin of the EMR signals of DNA declined without clear resolution.

Another important point to be emphasized here lies in the fact that most of the earlier discussions dealt with the EMR spectra of g-value of $c a .4$ and 2. The report by Müller $e t$ al. $=$ is unique in that their EMR spectrum for phage Tl shows the very broad peak at a low magnetic field. whose shape and position are very similar to the curve (c) in Figure 2 of the present study.

Blois et al..$^{33}$ made a thorough EMR study of DNA samples treated in many different ways. and they mentioned that there are four characteristic components to the DNA EMR spectra and one of them was "... a component centering near or at zero field with a tail which sometimes stretches several thousand gauss." They however. did not closely examine this particular component. which we believe is very important in regard to magnetic susceptibility of DNA. especially in dry A-state. They also concluded that the origins of the EMR signals from DNA are magnetic contaminations.

Recently, we showed for the first time. via EMR spectroscopy and SQUID measurements. that the double stranded natural salmon testes DNA in a dry state. or A-DNA. exhibits an intrinsic magnetism. ${ }^{14}$ In this context. the broad 
EMR line of the g-value greater than $10(\mathrm{~g}>10)$ was attributed to a possible mesoscopic ring current in ordered regions of the DNA that was responsible for the S-shaped magnetization in the magnetization-magnetic field strength (M-H) curve measured by SQUID. Whereas, the relatively sharp EMR peak of the g-value of about $2(\mathrm{~g} \sim 2)$ was ascribed to the Pauli spins existing in disordered amorphous regions producing linear magnetization. In particular, we conjectured that in a non-zero magnetic field the mesoscopic ring current might be driven by a circular motion of charge carriers along the helical $\pi$-ways including up to a few tens of parallel double stranded DNA (dsDNA) in the coherent tumneling regime. Surprisingly. we also learned that the broad EMR signal and the S-shaped magnetization behavior disappeared when the DNA samples were wet enough to be in the B-form.

Although the magnetism of $\hat{\lambda}$-DNA was already studied for the fully hydrated B-form in water solution by a French group ${ }^{12}$ led by Nakamae, the total magnetization in this case contains all the contributions from possible magnetic sources including the water solution and dissolved oxygen. Surprisingly, they claimed the $\lambda$-DNA samples in the wet B-form exhibited an unexpected paramagnetic uptum below $20 \mathrm{~K}$. which disappeared when the samples were dried. Mizoguchi et $\mathrm{al}^{2+}$ of Japan, however, argned that the paramagnetic results reported for $\lambda$-DNA in the B-state by the French group ${ }^{3:}$ might have been derived from oxygen molecules adsorbed by the DNA samples. In fact, the Japanese group ${ }^{3+}$ could even reproduce the French group's data by using the quartz wool containing adsorbed oxygen molecules. Nonetheless, we believe that the concept of the mesoscopic orbital paramagnetism put forvard by the French group is worth further, close examination. In order to further examine the conflicting reports and controversies as well as to sy stematically investigate the hydration dependence of magnetization of dsDNA, it is necessary to prepare samples by methods capable of ruling out the contribution from buffer components and oxygen. Therefore. in this work we further extend our investigation to study the dependence of magnetic properties of dsDNA on the degree of hydration via EMR spectroscopy and SQUID measurements.

\section{Experimental Details}

The salmon dsDNA consisting of 2000 base pairs was obtained from the Sigma \& Aldrich Chemicals (U. S. A.) and was utilized without any other treatment. We prepared different DNA samples at varying relative humidity by using the saturated salt method. which was used in the discovery of the helical structure of DNA by X-ray diffraction ${ }^{26}$ In order to obtain DNA samples with different water contents. they were first kept in a chamber for 4 weeks under varying humidity controlled via saturated salt solutions. ${ }^{26,37}$ Then. each dsDNA sample was placed in the gap between two separate poly styrene straws (Quantum Design Co.. U. S. A.). then loaded into $5 \mathrm{~mm}$ diameter quartz tubes (Wilmad glass. U. S. A.). Each DNA sample tube was dipped into liquid nitrogen. evacuated, and kept at $5 \times 10^{-5}$ Torr for 10 minutes. The DNA sample tube was then filled with helium gas after being wamed to room temperature and kept under a stream of helium for 5 minutes. This freeze-thaw cycle was repeated twice more. Finally, each DNA sample was dipped into liquid nitrogen for 5 minutes and the DNA sample tube was then sealed under $5 \times 10^{-5}$ Torr. The Absence of oxygen molecules in the samples was confirmed by their EMR spectra at room temperature. The most thoroughly dried sample was prepared as explained in the caption of Figure 2. For every EMR spectra we made corrections for the background signal.

EMR spectroscopy and SQUID magnetization measurements were employed to investigate the magnetic properties of the DNA samples, SQUID measures a bulk magnetization that is interpreted at the macroscopic level. whereas the EMR spectroscopic data can be analyzed at the molecular level. EMR spectroscopy was perfonmed using a Jeol JESFA200 X-band spectrometer $(9.1-9.5 \mathrm{GHz}$. Jeol, Japan). The DC magnetic field was swept from 0 to $10000 \mathrm{G}$ and the $\mathrm{AC}$ magnetic field modulation was applied at $5 \mathrm{G}$ amplitude with $100 \mathrm{kHz}$. The receiver gain used was $5 \times 10^{2}$. The experimental procedure was the same as in our previous study. ${ }^{14}$ The spin numbers for each EMR signals were estimated as described earlier. utilizing $\mathrm{CuSO}_{4} \cdot 5 \mathrm{H}_{2} \mathrm{O}$ as the reference. $^{1+}$

SQUID (mpms 7, Quantum Design. Co.. U. S. A.) magnetization data were collected at increasing temperatures. After all measurements were completed. we took each DNA sample from the quartz tube and then immediately performed a thermogravimetric analysis (TGA) to determine the water content. ${ }^{28}$ The mass difference was determined from the stepwise TGA thermogram by taking the mass at $170^{\circ} \mathrm{C}$ as a baseline. The baseline was obtained from the first heating (heating rate: $5^{\circ} \mathrm{C} / \mathrm{min}$ ) run under a continuous flow of $\mathrm{N}_{2}$ gas using a Mettler Toledo DSC $82 \mathrm{le}$ (Germany) differential scauning calorimeter.

\section{Results}

The EMR signals observed for the DNA samples did not originate from the absorbed oxygen molecules. The paramagnetic resonance lines in the range of $5000-10000 \mathrm{G}$ of oxygen molecules disappeared (see Figure 1) when the samples were treated by vacuum. as described in the Experimental Details section. The oxygen resonance peaks over 5000-10000 G are known to be induced by coupling between the rotational degree of freedom and spins $(S=1)$ of molecular oxy'gen. 29,30

Therefore. it is logical to assume that the EMR signals are associated only with inherent spin structures introduced to DNA molecules ${ }^{31}$ under plysiological conditions, during handling such as isolation and purification. or by the sample history such as exposure to different radiation sources (e.g. UV/Vis light). ${ }^{3-3.34}$ Although the most thoroughly dried sample was found to contain $28.7 \mathrm{ppm} \mathrm{Fe}$, this impurity level is so low that EMR signals for Fe would not be observed at room 


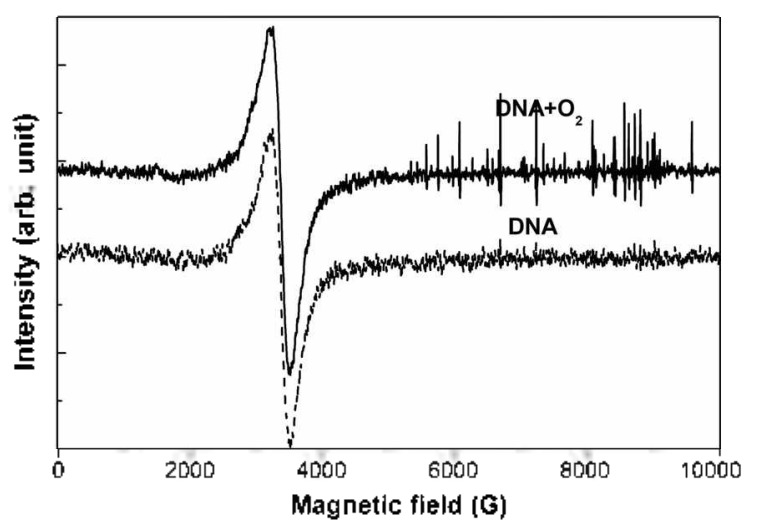

Figure 1. Comparison of EMR spectra obtained from a dsDNA sample in the absence or presence of oxygen molecules. The EMR peaks cansed by molecular oxygen are observed as very complex signals over the magnetic field range of $5000-10000 \mathrm{G}$.

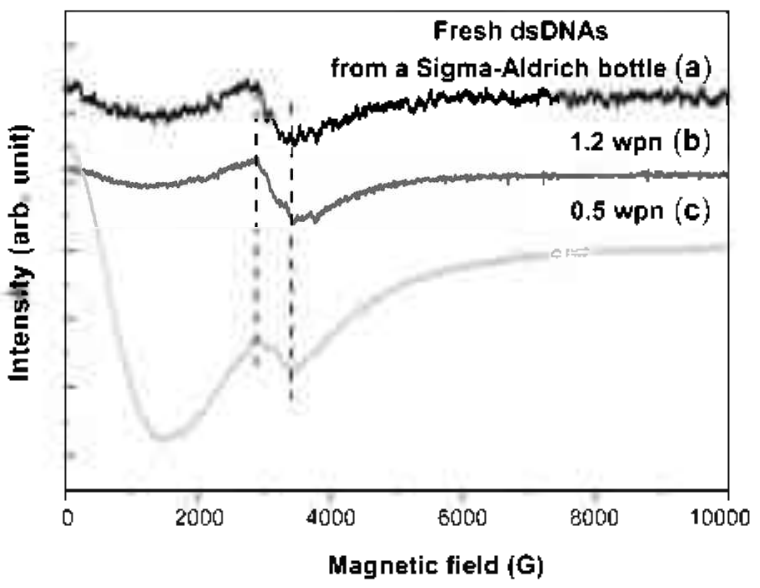

Figure 2. Dependence of typical EMR lineshapes on the sample history. The EMR spectrum (a) was obtained inmediately after a dsDNA sample was taken from a fresh reagent bottle (Aldrich \& Sigma). After the sample was kept for 2 months at $5 \times 10^{-5}$ Torr, the EMR spectrum (a) changed to spectrum (c). However, the signal (c) changed to spectrum (b) when the sample was reexposed to ambient humidity for $1 \mathrm{~min}$.

temperature.

In order to exclude the possibility that EMR signals at $\mathrm{g} \sim 2.0$ are not derived from the iron impurities. we intentionally added external $\mathrm{Fe}$ (III) into our DNA samples. But. no change in the signal shape and intensities were observed. This observation together with other researcher's reported results ${ }^{3.3 .35 .37}$ imply that the signals at $g \sim 2.0$ are not originated from iron impurities

Figure 2 shows typical EMR spectra as obtained at room temperature from a dsDNA sample that was exposed to varying relative humidity. The uppermost EMR signal (curve a) was obtained in the dry He-gas atmosphere for the DNA sample when freshly taken from the reagent bottle. The medium amplitude of the very broad EMR signal of $\mathrm{g}>10$. whose minimum appears at about $1500 \mathrm{G}$ was followed by another one of $\mathrm{g} \sim 2.0$ and 2.1, whose minimum is located at about $3500 \mathrm{G}$ and possesses a peak-to-peak distance or linewidth of $\Delta \mathrm{H}_{\mathrm{pp}} \sim 500 \mathrm{G}$ where $\Delta \mathrm{H}_{\mathrm{pp}}$ stands for the di- stance between the peak maxmum and minmum positions of the differential EMR spectrum. When the sample was quickly evacuated down to $5 \times 10^{-5}$ Torr and sealed in an EMR quartz tube ( $3 \mathrm{~mm}$ in diameter), significant variation in the EMR spectrum was not observed. However. the signal size of the two peaks was enlanced. as shown by the bottom spectrum (curve c) in Figure 2, when the sample was kept in the sealed sample tube for 2 months. Furthermore, the very broad EMR line of $g>10$ became significantly stronger when compared to the curve a shown in Figure 2. Interestingly enough, the two signals did not change over one and half years. When the dry sample was exposed to ambient humidity for $1 \mathrm{~mm}$, the signal $\mathrm{c}$ changed to the curve $\mathrm{b}$, which is very smilar to the curve $a$. The sample $b$ and $c$ were found to contain 1.2 and 0.5 water molecules per nucleotide unit (wpin), respectively.

It is important to note that the EMR signals observed for the dry samples are derived from spin species already existing within the dsDNA samples themselves and. in addition, their shapes and intensities do not change at room temperature. Moreover. the EMR lines do not exlubit any hyperfine structure. which is different from what would be expected for the spins localized at the atoms in the nucleotides. ${ }^{38.39}$ This spectral feature is completely different from the EMR results reported earlier by others ${ }^{21,33.33}$ for DNA samples whose spm species were intentionally generated by exposing them to radiation pulses, X-ray, and UV/Vis light, either in solution or in solid form at low temperature. Notably, Sevilla and coworkers ${ }^{\text {til }}$ pioneered the hole and electron transfer process in DNA after $\gamma$-irradiation at low temperature.

Shown in Figure 3 is a stack plot of EMR signals obtained for dsDNA containing varymg amounts of water. All the spectra were normalized by their mass after being corrected for the quality-factor $\left(\mathrm{Q}\right.$-factor) ${ }^{41}$ of the microwave cavity. First of all, the most dramatic variation in EMR signals observed is for the broad line of $g>10$. This peak is very strong when a DNA sample contains less than 1.0 water

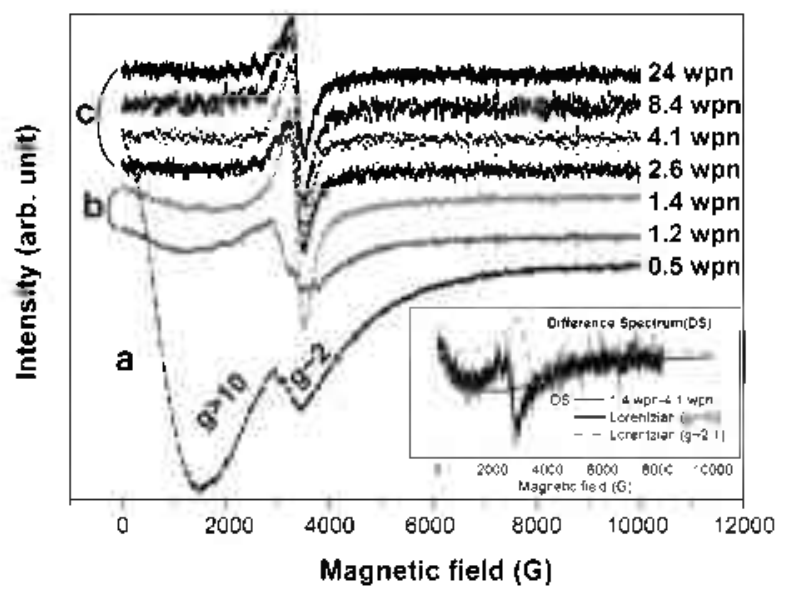

Figure 3. Schematic stack plots of the EMR signals obtained from dsDNA samples of varying water content. The inset shows the difference spectrum obtained from the spectra of DNA samples with 1.4 and $4.1 \mathrm{wpn}$. It also shows the deconvolution into two components. 
molecule per nucleotide unit (wpn) (see Figure 3a). It becomes much weaker (Figure 3b) for an A-DNA sample containing $1.2 \mathrm{wphl}$, and completely vanishes (Figure $3 \mathrm{c}$ ) when the DNA sample contains higher than $c a .2$ wpn despite the fact that the DNA samples are expected to be in the dry A-form at these levels of water of hydration. Needless to say. the B-DNA samples of higher water content do not show this peak.

If we closely inspect the lineshape of the EMR signals in Figure 3. the spectra can be largely divided into three groups. The first group (Figure 3a) contants the EMR spectrum obtained for the most thoroughly dried A-DNA. which is composed of an extremely broad and large EMR line of $\mathrm{g}>10$ appearing at $c a .1500 \mathrm{G}$ and another with a relatively narrow linewidth of $\mathrm{g} \sim 2.1$ (Figure 3a) appearing at $c a$. $3300 \mathrm{G}$ on which a very small peak of $\mathrm{g} \sim 2.0$ is superimposed. The second group (Figure $3 \mathrm{~b}$ ) includes for the ADNA samples containing limited amounts of water (1.2-2.0 wpn) revealing a broad EMR line of $g \sim 10$ with significantly reduced intensity together with two split lines of $\mathrm{g} \sim 2.0$ and 2.1 with slightly different linewidths $\left(\Delta \mathrm{H}_{\mathrm{pP}}=\right.$ 390 and $890 \mathrm{G}$ ) but appearing at quite close resonance fields. 3350 and $3200 \mathrm{G}$ The third group (Figure $3 \mathrm{c}$ ) contains the A-DNA samples containing higher amounts of water (2.6 wpn) and wet B-DNA samples that show a single narrow EMR signal of $g \sim 2.0$ with the broad peak of $g>10$ almost completely depressed or disappeared. Another peak of $g$ 2.1 is barely discenuble in this group, most probably due to extreme line broadening.

For the EMR peaks of the high water DNA samples (2.6$24 \mathrm{wpll}$. Figure $3 \mathrm{c}$ ), the single Lorentzian function was used to fit each EMR signal, sunce they were of very sumilar lineshapes and also showed only a single peak of $g \sim 2.0$. The lines were reasonably well fitted especially at the center of resonance. Accordungly, we carried out a simple double integration on each spectrum from group (c) in Figure 3 and obtained almost the same average EMR parameters of $\mathrm{g}=$ $\left.2.011( \pm 0.004), \Delta \mathrm{H}_{\mathrm{fw} w \mathrm{~h}}=618 \pm 38\right) \mathrm{G}$ and $3.2\left( \pm 0.3 \times 10^{16}\right.$ spins per gram nucleotide (spins/(g nucleotide)) independent of their water content. where $\Delta \mathrm{H}_{\text {f: }}$ him signifies the full width of the signals at half-maximum. This means that the EMR spin dynamics observed here are not govened by either dynamic fluctuations caused by the structural (i.e. conformational) degrees of freedom of the duplex DNA or by the environmental factors including water molecules. However. these dýnamics are dommated by an intrinsic motion of the spins themselves. It should be cautioned here that the number of spins estimated for the peak of $g \sim 2.0$ correspond to Curie spins. We can draw a better picture if we could estimated the number of Pauli spins..$^{22}$ Unfortunately. the density of states at the Fermi energy level of DNA is not known. which does not allow for us to convert the EMR peak intensity to the Pauli spins. The temperature-independent magnetic susceptibilities (Figure 7) of the DNA samples measured by SQUID strongly implies that the spins of the $\mathrm{g} \sim 2.0$ peak in our samples are of the character of Pauli spins. It is expected that the number of Pauli spins signifi- cantly exceed the number of Cune spins.

On the contrary. for the samples below $c a .2 .0$ wpn (i.e., those of 1.4 and $1.2 \mathrm{wpn}$ ) the EMR lineshape shows different patterns with a higher complexity; an additional two EMR peaks simultaneously appear at $\mathrm{g}-2.1$ and $\mathrm{g}>10$ together with the existing EMR signal of $g \sim 2.0$ with a much reduced relative intensity. Assuming that the EMR signals of $g \sim 2.0$ show the same lineshape but have different relative intensities, we tried to resolve all the EMR signals uto the line of $\mathrm{g}-2.0$ and the two others by subtracting the EMR spectrum [ $\mathrm{g}$ factor $=2.014\left( \pm 0.002, \Delta \mathrm{H}_{\text {fwitun }}=577\right.$ $( \pm 19) \mathrm{G}]$ of the 4.1 wpn DNA sample. taken as a representative quasi l-D component, from the total spectrum of the DNA sample containing 1.4 wpn. The lineshape of the separated difference spectrum (DS) (inset in Figure 3) closely resembles that of the EMR spectrum of $1.2 \mathrm{wpn}$, and the two peaks of $g>10$ and $g \sim 2.1$ were well deconvoluted into two Lorentzian functions.

The spectral parameters thus obtained are compared $\mathrm{m}$ Figure 4. Figure ta shows the dependence of the linewidth of the three peaks of $g>10.2 .1$ and 2.0 on the content of water in the DNA samples. The first peak of $g>10$ greatly broadens with increasung water content. whereas the linewidth of the peak of $\mathrm{g} \sim 2.0$ remains almost constant. The peak of $g \sim 2.1$ tends to decrease slightly in linewidth with uncreasing water content. Figure $4 \mathrm{~b}$ compares the magnetic
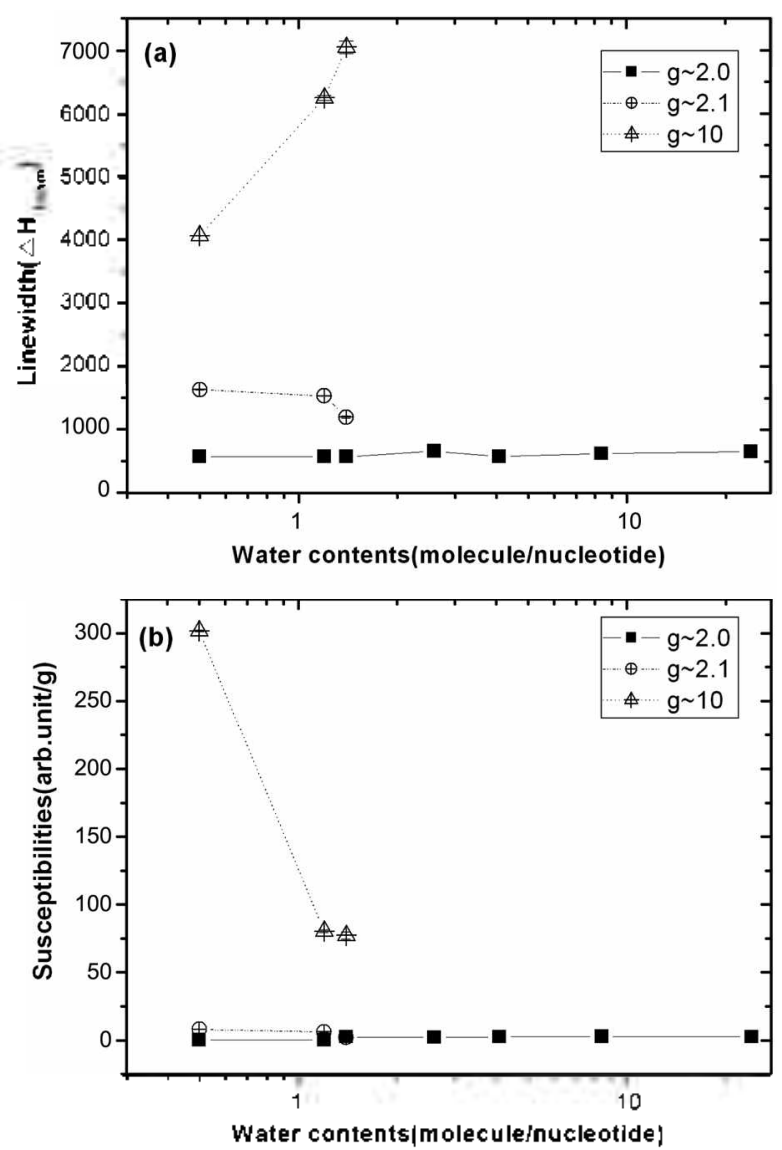

Figure 4 . The dependence of linewidth (a) and susceptibility (b) of the EMR signals on water content at room temperature. 
susceptibility dependence of the three peaks on the water content. The susceptibility values were obtained by double integration of resolved peaks. The magnetic susceptibility. thus the g-value of the peak of $\mathrm{g}>10$, dinunishes sharply with mcreasing water content. whereas those of $g-2.1$ and 2.0 exhibit much less dependence on water content. The spin numbers estimated for the peak of $\mathrm{g}>10$ for the most dry sample (Figure 3c) is $4 \times 10^{18}$ spins/gnucleotide, while those for the combined peak of $g \sim 2.1$ and 2.0 ranged from about $2 \times 10^{16}$ to $1 \times 10^{17} \mathrm{spins} / \mathrm{g}$.nucleotide.

It should be noted that natural dsDNA sanples are known to show a wide range of conformational phases according to their water content $t^{60.28,+3,+4}$; the hydrated B-form results above $20 \mathrm{wphl}$, a mixture of phases consisting of the A- and B-forms at $8-20 \mathrm{wpn}$. the A-form with a certain degree of conformational freedom at 4-8 wpn. and the dry A-form without much conformational freedon below 4 wpln.

In order to further understand how such morphological changes would influence the result of our study. we tried to relate the EMR lineshapes to motional dumension of the spins involved. In the case of slow motional spins $\left(\omega \cdot \tau_{c}\right\rangle$ 1 , (1): resonance angular frequency, $\tau_{c}$ : characteristic correlation time). the EMR lineshape is expected to be of pure Gaussian by the central limit theorem. On the contrary, in the limit of fast motional regime $\left(0 \cdot \tau_{c} \ll 1\right)$, the lineshape is satisfactorily described by the Lorentzian function due to the three-dimensional isotropic motional average effect. However. If the spin species in the fast motional regime are confined withun a reduced dimension, the motional average would lead to the EMR lmeshape satisfying the Lorentzian function at the central resonance part but would lead to the Gaussian-like lineshape in the tail part far away from the central resonance point (refer to the inset in Figure 5). ts $^{\text {s. }}$ Accordingly, as in organic conducting polymers or low dimensional magnetic systems, the motional dimension of carrers or spins can be analyzed simply by plotting $((\mathrm{H}$ $\left.\left.\mathrm{H}_{\mathrm{res}}\right) / \Delta \mathrm{H}_{12}\right)^{2}$ ws $\mathrm{I}\left(\mathrm{H}_{\mathrm{res}}\right) / \mathrm{I}(\mathrm{H})$, where $\mathrm{H}$ and $\mathrm{H}_{\mathrm{res}}$ are applied and resonance magnetic fields. $\Delta \mathrm{H}_{1: 2}$ the half width at the half maximum. and $\mathrm{I}\left(\mathrm{H}_{\mathrm{res}}\right)$ and $\mathrm{I}(\mathrm{H})$ indicate amplitudes at the

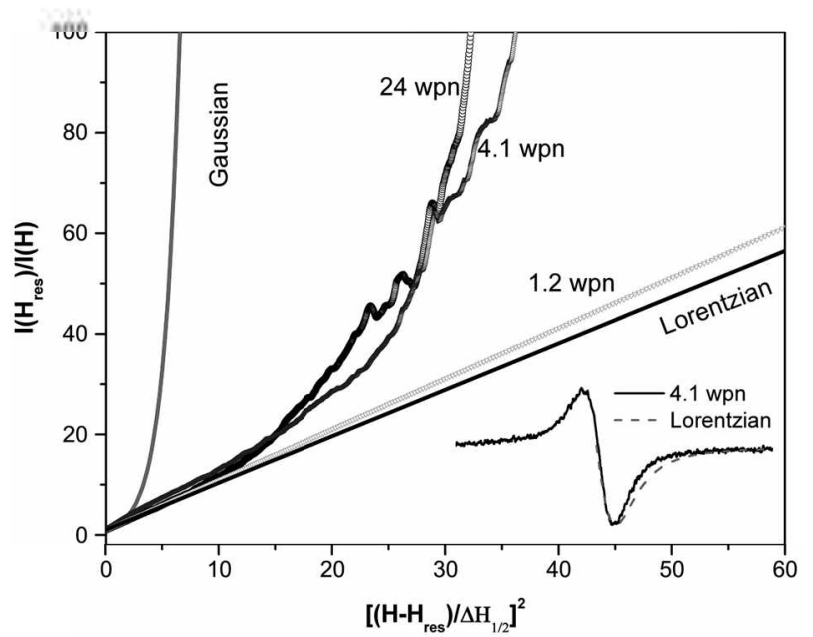

Figure 5. The motional dimension analyses of spins vid $\left[\left(\mathrm{H}-\mathrm{H}_{\mathrm{Te}}\right)\right.$ $\left.\Delta \mathrm{H}_{1: 2}\right]^{2}$ vs. $\mathrm{I}\left(\mathrm{H}_{\mathrm{res}}\right) \mathrm{M}(\mathrm{H})$ plots. resonance point and applied magnetic field, respectively.

Figure 5 demonstrates such plots for the EMR peaks containing both g-values of 2.1 and 2.0. It is apparent that the plots for wet DNA samples (4.1 and 24 wpn). which show mainly the peak of $\mathrm{g} \sim 2.0$, are occupying a central region between the Gaussian and Lorentzian predictions implying that charge carriers are involved in quasi one dimensional (I-D) motions. As a result. we conjecture that the spins experience quasi 1-D diffusive motion along the long axis of the double helis when the water content lies in the range of the $2.6-24 \mathrm{kpn}$. This is compatible with a number of experimental reports by several research groups, ${ }^{1 \cdot 3.45}$ including Barton $e t$ al. ${ }^{47.50}$ who pioneered the charge transfer/transport in B-form DNA. The research groups of Giese, ${ }^{\text {,l }}$ Saito. ${ }^{52}$ Schuster, ${ }^{5,54}$ and Lewis and Wasielewski ${ }^{5,56}$ also made important contributions to the understanding of longrange charge transport mostly in B-DNA samples.

From the same dimensional analysis for the peak of $g$ 2.1 of the DNA sample with $1.2 \mathrm{wpn}$, we know that the spins of this peak are well described by a three-dimensional (3-D) motion because the $\mathrm{I}\left(\mathrm{H}_{\mathrm{res}}\right) / \mathrm{I}(\mathrm{H})$ is $\left(\left(\mathrm{H}-\mathrm{H}_{\mathrm{res}}\right) / \Delta \mathrm{H}_{1: 2}\right)^{2}$ plots show a straight line (i.e, the line for the DNA sample of 1.2 wpn and 1.4 wpn in Figure 5) perfectly matching Lorentzian behavior. As a result. it is safe to say that the charge camers are likely to move along single DNA double strands with relatively high water content, but that the spin motion either gradually or abruptly becomes three-dimensional (i.e. from quasi l-D to 3-D) with decreasing water content. In short, the two EMR lines of $g \sim 2.1$ and $g \sim 2.0$ have Lorentzian and mid-Lorentzian-Gaussian lineshapes reflecting the threeand quasi one-dimensional motion of spin species, respectively. Recently. Mizoguchi and coworkers ${ }^{57}$ also made similar observation on the dimensional dependence of magnetic properties of Mn-DNA samples on water content.

It should again be pointed out that the samples revealing a broad signal of $\mathrm{g}>10$ are always accompanied by the peak of $\mathrm{g} \sim 2.1 \mathrm{in}$ addition to the peak of $\mathrm{g} \sim 2.0$ with much

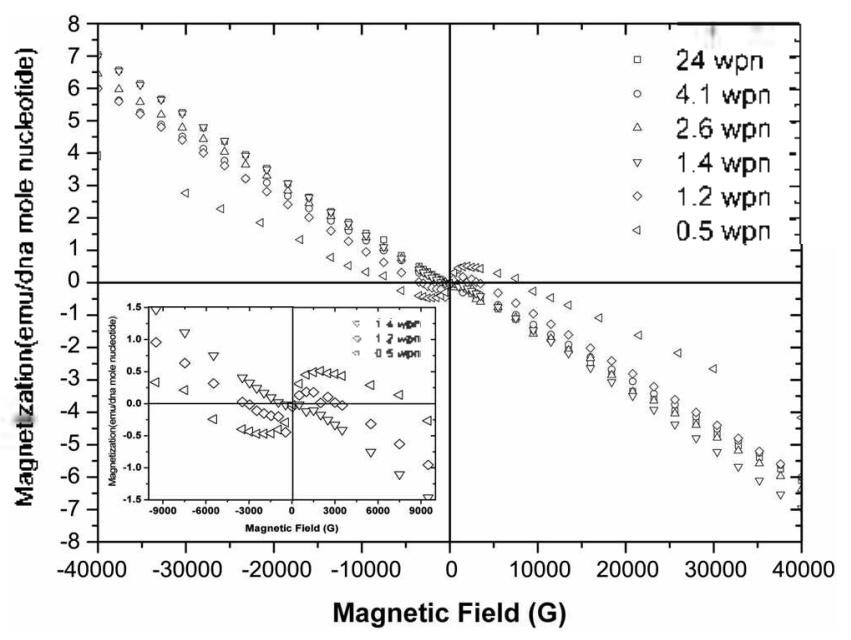

Figure 6. The magnetization-magnetic field strength $(\mathrm{M}-\mathrm{H})$ curves obtained at room temperature for the dsDNA samples with different water contents. The inset shows the magnified central sshaped portions of the M-H curves. 
reduced intensity. As described above, the latter appears for all the samples regardless of the water content. In our earlier report, we overlooked the fact that the peak at about $3500 \mathrm{G}$ consists of two separate peaks of $\mathrm{g}$-values of $c a .2 .0$ and 2.1.

The magnetization-magnetic field $(\mathrm{M}-\mathrm{H})$ curves (Figure 6) for DNA samples containing different levels of water were obtained via SQUID magnetic measurements. It is noted that the DNA samples containing 2.6-24 wpn and exhibiting mainly the EMR signal of $g \sim 2.0$ show linear diamagnetic $\mathrm{M}-\mathrm{H}$ lines over the whole range of the magnetic field studied. Whereas those containing less water ( 1.4 wpnn) and showing simultaneously the EMR signals of $g>10$. $\mathrm{g} \sim 2.1$ and $\mathrm{g} \sim 2.0$ exhibit both an $\mathrm{S}$-shaped magnetization behavior in the low field region and a linear dependence of magnetization outside this region.

The inset in Figure 6 clearly demonstrates that the amplitude (i.e. the difference between the maximum and minmum points) of the S-shaped part of the $\mathrm{M}-\mathrm{H}$ curve continuously dinunishes as the water content in the DNA samples increases. Eventually, the S-shape completely disappears at the water content of $c a .2 .6 \mathrm{wpn}$. It should be noted that the $\mathrm{S}$-shaped portion of $\mathrm{M}-\mathrm{H}$ curves consists of the paramagnetic ascending part followed by the diamagnetic descending part.

The magnetic susceptibility-temperature $(\chi-T)$ curves measured at 1000 and $10000 \mathrm{G}$ are given in Figure 7. We corrected the raw data by subtracting the diamagnetic contribution $^{14}\left(-2.71 \times 10^{-4} \mathrm{emu} / \mathrm{G}\right.$ mol nucleotide $)$ by the DNA molecule itself and also the same $\left(-0.72 \times 10^{-6} \mathrm{emu} / \mathrm{G} \cdot \mathrm{g}\right)$ by water. ${ }^{\text {is" }}$ The diamagnetic contribution of DNA was estimated by the so-called Pascal's constants of each element and chemical bonds. ${ }^{\text {is }}$

The two magnetic fields were specifically chosen because they each correspond to the para- and diamagnetic regions of the S-shaped portion of the $\mathrm{M}-\mathrm{H}$ curves given in Figure 6. The $\chi$-T curves shown in Figure 7 reveal several important generalities: 1) All the magnetic susceptibility $(\mathcal{X})$ values are positively indicating that the electronic structure of DNA

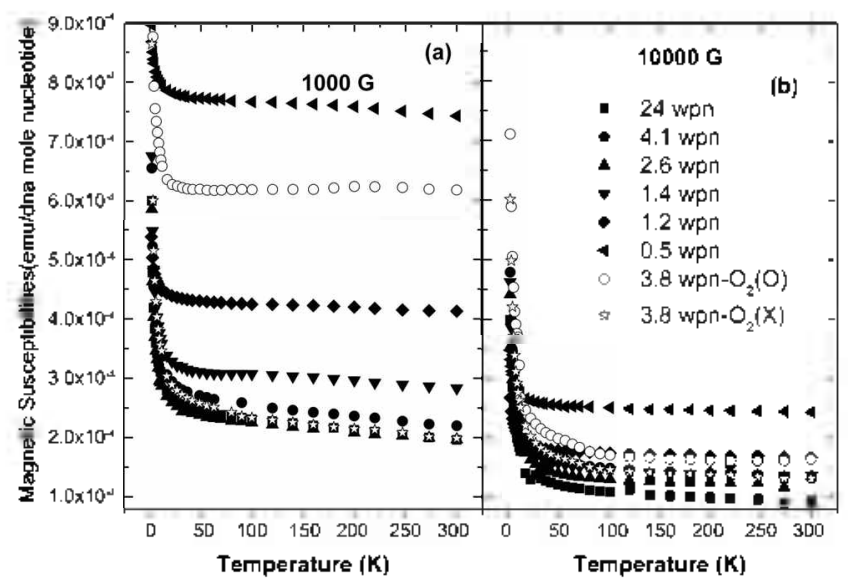

Figure 7. Molar susceptibilities vs. temperature $(\chi-T)$ curves observed at (a) $1000 \mathrm{G}$ and (b) $10000 \mathrm{G}$. The data for a sample with 3.8 wp containing oxtgen molecules ( $2 \mathrm{ppm}$ ) are compared with those for a deoxygenated sample. results in an inherent paramagnetism. 2) The magnitude of $\mathcal{X}$ value increases steadily as the water content in DNA samples decreases. The $\chi$ value $\left(7.8 \times 10^{-4} \mathrm{emu} / \mathrm{G}\right.$ mol nucleotide $)$ at $1000 \mathrm{G}$ of the DNA sample containing $0.5 \mathrm{wpn}$ is about four times the value $\left(2.1 \times 10^{-4} \mathrm{emu} / \mathrm{G} \cdot \mathrm{mol}\right.$ nucleotide $)$ of the wet DNA in the B-form ( 24 wpr). 3) The $\chi$ value changes very little from room temperature to $50 \mathrm{~K}$.4) All the compositions exhibit an upturn in the $\chi$ value around $5 \mathrm{~K}$, most probably due to the presence of a small amount of magnetic mpunities such as iron. 5) The $\chi$ values at $1000 \mathrm{G}$ are significantly higher than the corresponding values at 10000 $\mathrm{G}$

Needless to say, the presence of oxygen molecules in a sample will result in higher magnetization or magnetic susceptibility values as shown by the curves given in Figure 7. As one can see from Figure 7. the magnetic susceptibility value of the particular DNA sample containing $3.8 \mathrm{wph}$ $\left(\mathrm{H}_{2} \mathrm{O}\right)$ and $1.9 \times 10^{9}$ molecules of oxygen $\left(\mathrm{O}_{2}\right)$ in the sample tube of $1.4 \times 10^{-6} \mathrm{~m}^{3}$ is $c a .2 .5 \times 10^{-4} \mathrm{emu} / \mathrm{G}$ mol nucleotide at $10000 \mathrm{G}$ and $c a .6 \times 10^{-4} \mathrm{emu} / \mathrm{G} \mathrm{mol}$ nucleotide at 1000 $G$ which is significantly higher than those for the deoxygenated wet sample $(3.8 \mathrm{wpn})$. This was part of the recent controversial point between the French ${ }^{1,2,5}$ and Japanese groups. ${ }^{24}$

\section{Discussion}

The dependence of the EMR spectrum at room temperature on the water content. shown in Figure 3, clearly demonstrates that the EMR lines of g-values of 2.0,2.1 and greater than 10 did not onginate from impunties. Earlier, we argued that only at low temperatures could we observe the appearance of the EMR peaks for Fe(III), whose content in the dry sample was about $30 \mathrm{ppm} .{ }^{14}$ One may ascribe the hydration effect to the presence of ferromagnetically interacting impurities. These impurities can be separated by water molecules resulting in a superparamagnetic behavior. This possibility is excluded in the present case by the fact that the average distance between iron impurities is estimated to be $20 \mathrm{~nm}$. which is too far a distance for ferromagnetic coupling between the impurities to occur. ${ }^{\text {i. }}$

Mesoscopic understanding of carrier's interduplex tunneling. As presented earlier, ${ }^{14}$ we again propose that the broad EMR line of $\mathrm{g}>10$ and the S-shaped $\mathrm{M}-\mathrm{H}$ curve observed for oxygen-free A-DNA are attributed to the mesoscopic ring current induced by an external magnetic field. If this is indeed the case the intensity of the peak of $g>10$ should correlate to the magnitude of the induced mesoscopic ring current. ${ }^{(1)}$ As described in the previous section. the broad EMR line and the S-shaped magnetization of the DNA samples rapidly decrease with increasing water content. In fact. even a very low water content (greater than only 2.6 wpn) almost completely suppresses the broad EMR line of $\mathrm{g}>10$ and the S-shape in the middle of the $\mathrm{M}-\mathrm{H}$ curve. This strongly implies that the water molecules efficiently disturb the inesoscopic ring current. Such an S-shaped behavior is generally observed for so-called type II superconductors. We 
are not yet sure if there exists any correlation between this observation and the claim by Kasumov et al ${ }^{61}$ that DNA may be a proximity superconductor.

Consistent with present observations. our proposed picture for the difference between the carrier's transport in dry (ADNA) and wet DNA (B-DNA) samples is that the dsDNA samples in a dry state experience mesoscopic ring currents in the ordered regions, as conjectured earlier by us. This implies that there is a helical transport along the stacked- $\pi_{z}$ of the helical structure as well as perpendicular coherent transport achievable only by efficient lateral tumeling resulting in the formation of ring current. In this case. the induced magnetization can be strongly enhanced owing to their positive interference effect. However, if the coherence and helicity of the charge transport were disrupted by a certain external agent or disturbance resulting in diffisive transport. the induced magnetization would diminish. In this comection. Tagani et al..$^{\circ 2}$ theoretically demonstrated that electronic transport of benzothiophene-based chiral molecular wires can be interpreted not only by taking the polymer chains as molecular solenoids but also by the applicability of the current-induced magnetic field to such molecular solenoids.

In other words, in order to account for the three-dimensional motion of spins in addition to their quasi one-dimensional motion. interduplex spin migration mechanisms such as tumneling or magnetically induced interduplex synchronized motions have to be adopted. In fact. the possibility of interduplex tunneling has been discussed by Sevilla et $\mathrm{al}^{63}$

One convincing example for this picture can be found in carbon nanotubes ${ }^{6-3}$ with helical current around their tube axis under non-zero magnetic fields. Some of the earlier studies on carbon nanotubes experimentally showed the appearance of broad EMR signals, sinilar to ours, as well as the externally induced magnetization comparable to $10 \mu B$. where $\mu B$ stands for the Bohr magneton. The nanotubes. however. do not show any S-shaped magnetization behavior because no lateral tunneling is possible between the nearest carbon nanotubes. In the case of dsDNA. the stacked- $\pi_{2}$ orbitals are expected to play an important role in the aforementioned coherent helical charge transport. In addition. one of possible mechanisms is that dsDNA in the ordered regions allows a coherent lateral interduplex tunneling to occur throughout the elementary fibrils that have dimensions of $c a .60 \mathrm{~nm}^{1+4}$ and show liquid crystalline birefringence on a cross-polarizing microscope (Figure 8). Formation of liquid crystalline (LC) phases by DNA was thoroughly reviewed by Livolant and Leforestier. ${ }^{6 *}$ and recently Smalyukh et $a l^{60}$ discussed LC pattern formation in drying droplets of DNA. Lornan $e t a l^{67}$ and Manna $e t a t^{68}$ also recently reported many new crystalline and non-conventional phases of DNA. We believe that the water content is expected to control much of the liquid crystalline morphology of DNA. which. in turn. would influence the magnetic properties. Therefore. and exact understanding of the molecular order under different hydration enviromment can be useful and important. This aspect requires further studies

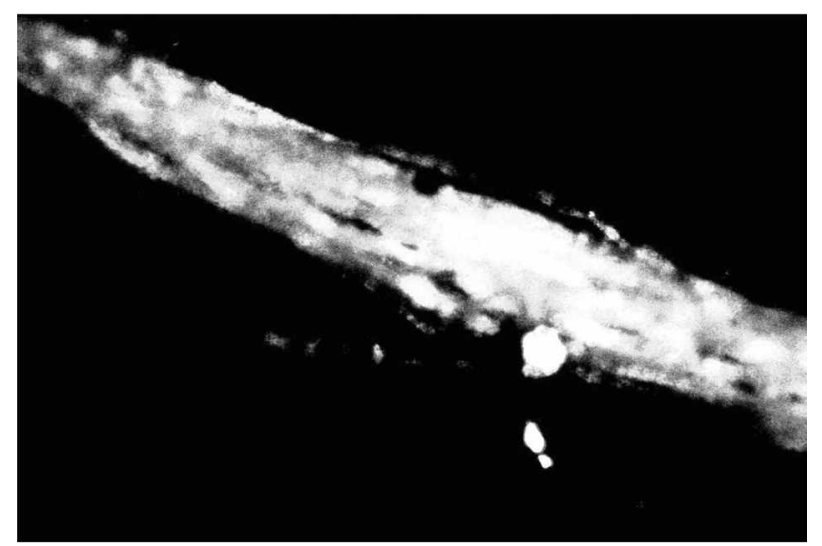

Figure 8. Biretringence of a DNA fibril observed through a cross polarizing optical microscope at room temperature (magnification $200 \mathrm{X}$ ).

Therefore. the intensity of the broad EMR line should be proportional to the number of duplexes experiencing colerent lateral transport and be reflected in the S-shaped $\mathrm{M}-\mathrm{H}$ curves obtained by SQUID measurements. Based on this conjecture, one can envisage that diminution in the coherent lateral tunneling between duplexes may be responsible for the weakened broad EMR lines and the S-shaped magnetization for the DNA samples containing water molecules. In other words, although each dsDNA can experience a helical current flow. if the helical currents do not laterally couple colherently in phase. the broad EMR line and the S-shaped $\mathrm{M}-\mathrm{H}$ curve weaken and finally disappear due to destructive interference by water molecules. The water molecules present in dsDNA are expected to play the role of effective scattering centers for spin motions. Since the higher water content leads to an increased number of scattering centers, the EMR linewidth may be broadened and finally the peak of $g>10$ will disappear with increasing water content. as we observed in the present study. Sevilla and coworkers ${ }^{6 z}$ also concluded earlier. via EMR studies of $\gamma$-irradiated DNA samples. that the DNA hydration layer can separate the duplexes and thus retard interduplex transfer of charge carriers. On the other hand. Kornyshev et $a l^{\text {ti }}$ claimed from $\mathrm{X}$-ray diffraction studies that strong azimuthally dependent interactions between neighboring DNA molecules persist to ca. $20 \AA$ surface-to-surface separations.

However. pure tumeling alone is not enough to explain the mesoscopic ring current occurring over $60 \mathrm{~nm}^{14}$ of the lateral size of dsDNA fibrills since the tumeling probability diminishes exponentially with distance. Moreover. a phasecoherent transport must occur with difficult throughout the regime. In order to overcome this complicated situation. we propose a cyclotron motion along the inherent helical $\pi$-ivay of dsDNA in the non-zero magnetic field. This concept, however, requires further tests. such as the measurement of the dependence of carriers transport on the frequency of an external field. In this context the discussion by Mesquita et $a h^{71}$ on large-scale quantum effects in biological systems appears to be an important theoretical contribution to the understanding of this proposition. They are paying attention 

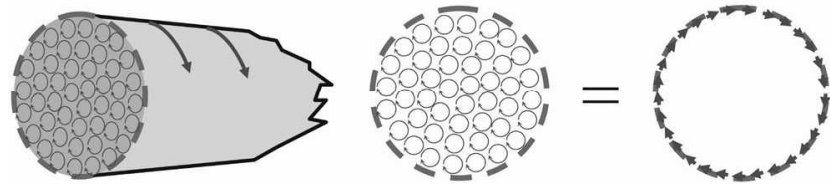

Figure 9. An idealized picture of the cyclotron motion of spin carriers in an elementary fibril of dsDNA. The inner small circles designate the solenoidal motions along dsDNA.

to the possible large-scale colnerent process, which would allow long-distance transport of signals in nonequibilium biopolymer systems. In particular, they rely on the so-called Frohlich effect. The view expressed recently by Sension ${ }^{71}$ on the quantum path to photosynthesis also emphasizes the importance of coherence energy transfer or electronic coherences in complex biological sy stenis.

From molecule to mesoscopic phase. In the presence of a magnetic field the Lorentz force causes the electrons to move along circular or helical paths. Such a periodic motion is called cyclotron motion. If there are free charge carriers in the stacked- $\pi_{z}$ orbital, then they experience cyclotron motion along the dsDNA's inherent helical $\pi$-way including the stacked- $\pi_{z}$ orbital. The cyclotron radius increases with increasingly applied magnetic field and finally matches the radii of helical $\pi$-way of dsDNA. The cyclotron resonance $(\mathrm{CR})^{7 \hat{2} \cdot 74}$ is expected to occur when the cyclotron frequency $\mathrm{u}_{\mathrm{s}}$ at the matching magnetic field becomes the same as the external angular frequency $(o)$ and thus observable in the linit of $\omega) \tau_{k}>\mathrm{I}\left(\tau_{k}\right.$ : momentum scattering time $)$.

From the resonance condition of $\omega=\omega_{c}=v_{-} / r=e H_{r} / m^{*} c$ for a single A-dsDNA (where $r$ is the radius of A-dsDNA and $(1)$ the EMR spectrometer frequency). one can calculate the circumferential velocity of $v_{\perp}=r \omega=1.32 \times 10^{-7} \mathrm{~cm} \times 2 \pi$ $\times 9.45 \times 10^{9} \mathrm{~s}^{-1}=8 \times 10^{3} \mathrm{~cm} / \mathrm{s}$. Also. the longitudinal velocity along the helical axis of $v_{1}=3 \times 10^{3} \mathrm{~cm} / \mathrm{s}$ is obtained because the spin carriers have to go through one helical pitch of $28.2 \mathrm{~A}$ for A-DNA during the same period of time $\mathrm{T}=\mathrm{l} / \mathrm{v}$ $\left(v=9.45 \times 10^{9} \mathrm{~s}^{-1}\right)-1.06 \times 10^{-16} \mathrm{~s}$. These values are of the same order of magnitude as those reported for typical semiconductors. ${ }^{75.77}$ It also should be noted that the values of $v$ and "are of the same order.

The coherent helical motion (Figure 9) of spin carriers at very low levels of water content can exert a mutual inductive effect on to neighboring DNA helices according to Faraday's law $^{75}$ in electromagnetism. As a consequence, a carrier motion can crossover from a quasi one-dimensional regine to a coherent three-dimensional regime. which would eventually realize a large lateral loop current throughout ordered regions.

\section{Conclusion}

We explored the hydration effect on the magnetic properties of dsDNA by relying on EMR spectroscopy and SQUID magnetometer measurement. As the water content was lowered. the magnetic properties showed a transition-like behavior below $c a$. 2 wpn. For the higher water content (2.6-
24 wpn). the EMR spectrum resulting from a Zeeman transition shows mainly a single peak of $g \sim 2.0$ that is describable in terms of the quasi one-dimensional motion of spins.

Correspondingly, the SQUID magnetization measurement also displays linear diamagnetic $\mathrm{M}-\mathrm{H}$ relations, for example, with $\chi-1.1 \times( \pm 0.5) 10^{-4} \mathrm{emu} / \mathrm{G}$ (mole nucleotide) for dsDNA containing $24 \mathrm{wpn}$. On the contrary, below 2 wpn, the EMR spectra are composed of a newly appeared broad peak of $\mathrm{g}>10$ and a relatively sharp, additional Zeeman transition peak of $g \sim 2$.l together with the much suppressed EMR signal of $g \sim 2.0$ also observed for the samples containing higher levels of water. The broad EMR lines of $g>10$ are due to the cyclotron resonance absorption associated with an electric dipole transition of spins with charge. and these can be mediated by an interaction between the charge carriers and oscillating microwave magnetic field wia so-called spinorbital coupling along the helical $\pi$-wway of dsDNA under non-zero magnetic fields. When the peak of $g>10$ appears together with the peak of $g \sim 2.1$, the latter can be understood in terns of Zeeman transition of spin species experiencing cyclotron motion. Accordingly, we can conclude that the spins of $g \sim 2.1$ are located in ordered regions participating in the spin-orbital coupling. On the other hand the spins of $g-2.0$ undergoing Zeeman transition are confuned in the disordered region excluded from the spin-orbital interactions.

Moreover, the increasing S-shaped component of the M-H curves in SQUID measurements with decreasing water content can be ascribed to Faraday's nutual magnetic induction between the nearest neighbor's dsDNA. This picture allows us to assume a few tens of nanometers of mesoscopic ring currents occur. which is essential in our interpretation of the magnetic properties of A-dsDNA. The sensitivity of DNA's magnetic properties to water content also must be related to structural changes caused by the water molecules even at low lydration levels.

All the observations discussed above on the influence of water on the magnetic properties of DNA warn us that whenever we study the magnetic properties of natural as well as modified DNA, we should control the water content in the samples. In addition. we learned during this investigation that the DNA samples can be more readily contaninated with dissolved oxygen when they are wet than when they are dry.

Consequently. the present investigation enables us not only to clarify the cause of the magnetisn attributed to the structural characteristics of dsDNA. but also to the understanding of magnetic properties of hỵdrated DNA.

Acknowledgments. This research was supported by the Korea Science and Engineering Foundation through the Center for Electro- and Photo-Responsive Molecules. Korea University. The authors would like to express sincere thanks to N. Ogata and J. Grote for their advice and support. YoungWan Kwon. Eui-Doo Do, and Jun-Sung Kang were recipients of the Brain Korea 21 fellowship provided by the Ministry of Education and Human Resources. Korea. 


\section{References}

1. Long-Range Charge Transfer in DNA I: Schuster. G. B. Ed: Springer: Heidelberg. 2004.

2. Long-Range Charge Transfer in DNA H: Schuster. G. B.. Ed: Springer: Heidelberg. 2004.

3. Charge Transfer in DNA: From Mechanism to Application: Wagenkinedit H.A. Ed. Wiley-VCH: Verlag. 2005.

4. Kim. H.: Lee. M.: Sim. E. Bull Korean Chem. Soc. 2007, 28.607.

5. Watanuki. A.: Tkeda. H.: Yoshida. J.: Ogata. N. Kobmoshi Ronbunshit 2006. 63.419.

6. Grote, J. G.: Diggs, D. E.; Nelson, R. L.: Zetts, J. S.: Hopkins. F. K.: Ogata, N.: Hagen. I. A.: Heckman, E.: Yaney. P. P.: Stone. M. O.: Dalton. L. R. Hol Chinst Liq. Const. 2005. +26.3

7. Steckl. A. J. Kat. Photonics 2007, 1.3

8. Lee. J. M.: Choi J. Y.: Kim. J. M.: Lee. S. Y.: Lee. H.: Kim. S. K.: Cho. T. S. Bull. Korean Chent Soc. 2007. 28.965.

9. Bliumenfeld. L. A. Biofizika 1959, 4. 515.

10. Shulman, R. G.: Walsh Ir, W. M.: Williams, H. I.: Wright I. P. Biochem. Biophns. Res. Conmm 1961. 5. 52

11. Walsh Jr. W. M.: Shulman. R. G.: Heidenriech. R. D. Katue 1961. 16. 1041 .

12. Nakamae. S.: Cazayous. M.: Sacuto. A.: Monod. P.: Bouchiat. H. Phys. Rev. Lett. 2005. 94. 248102.

13. Mizoguchi. K: Tanaka, S.: Ogawa. T.: Shiobara. N.; Sakamoto, H. Phys. Rev. B 2005. $72,033106$.

14. Lee. C. H: Kwon. Y.-W: Do. E.-D.: Choi. D.-H.: Jin. J-I.: Oh. D.-K.: Kim. J. Phns. Rev B 2006. 73. 224417.

15. Mirkin. C. A.: Lestinger. R. L.: Mucic. R. C.: Storhotf. J. J. Nature 1996, 382,607

16. Gazit E. FEBS J. 2007.274.317.

17. Alivisatos. A. P.: Johnsson, K. P.: Peng. X.: Wilson. T. E.: Loweth. C. J.: Bruchez Jr. M. P.: Schultz. P. G. Vattwe 1996. 382.609.

18. Park. S. Y.: Lee. J.-S.: Georganopoulou. D.: Mirkin. C. A.: Schatz. G. C. J. Phis. Chem B 2006. 110. 12673.

19. Richter J. Phsica E 2003, 16, 157.

20. Gords W. Pruden, B.: Snipes. W. Proc. Natl Acad Sci. U.S.A. $1965,53,751$.

21. Sheng. P. K.: Bliumenfeld. L. A.: Kalmansen. A. E.: Pasynskii. N. G. Biofizika 1959. 4.263.

22. Muller. A.: Hotz. G.: Zimmer. K. G. Biochem. Biophns. Res. Conmmin. 1961 4. 214

23. Blois J, M. S.: Maling J. E. Kaskotich. L. T. Biophns. J. 1963, 3. 275

24. Mizoguchi. K.: Tanaka. S.: Sakamoto. H. Plns. Rev Len. 2006. 96.089801

25. Nakamae. S.: Cazayous. M.: Sacuto. A.: Monod. P.: Bouchiat. H. Plys. Rev. Lett. 2006. 96.089802.

26. Franklin. R. E.: Gosling. R. G. Acta Cnyst. 1953, 6.673.

27. OBrien F. E. M. J. Sci. hishtum. 1948. 25.73

28. Lee. S. L.: Debendetti. P. G.: Errington. J. R.: Pethica. B. A.: Moore. D. J. J. Phws. Chent. B 2004. I08. 3098.

29. Tithlham. M.: Strandberg. M. W. P. Phns. Rev: 1954.97. 951

30. Yagi M.: Takemoto, S.: Sasabe. R. Chem. Lett 2004. 33, 152.

31. von Sonntag. C. Freeradical-Induced DNA Damage and Its Repair: At Chemical Perspective? Springer: Heidelberg. 2006.

32. Shields. H.: Gordy. W. Proc Natl .Acad Sci LS.A. 1959. t5. 269

33. Ehrenberg. A.: Ruppercht. A.: Strom. G. Science 1967. 157. 1317.

34. Sevilla. M. D.: Becker. D. ESR Shudies of Radiation Damage to DNA and Related Biomolecules in Electron Paramagnetic Resonance' Gilbert. B. C.. Davies, M. I.. Murphy, D. M.. Gilbert, B. C.. Davies. M. J. McLauchlan. K. A.. Eds.: RSC Publishing: 2004: Vol. 19. 243

35. Iiyama. T.: Chikira. M.: Oyoshi. T.: Sugiyama. H. J. Biol. Inorg. Chem. 2003, 8. 135.

36. Castner, T.: Newell. G. S.; Holton, W. C.; Slichter. C. P. J. Chem.
Phss 1960.32.668.

37. Kliava. T. Phts. Stat Sol (b) 1986. 134.411

38. Nicolau. C. Shot-lived Free Radicals in Aqueons Solutions of Nucleic Acid Components in Phsico-chemical Properties of Nucleic Acids: Duchesne. J.. Ed.; Academic Press (London): London. 1973; Vol. 1. p 143.

39. Herak. T. N. E. p. $r$ of Irradiated Singte Crnstals of the Nucleic Acid Constituents in Phosico-chemical Properties of Nucleic Acids: Duchesne. J. Ed: Academic Press (London): London. 1973: Vol. 1, p 197

40. Cai. Z.: Sevilla. M. D. Studies of Excess Electron and Hole? Transfer in DNA at Low Temperatwes in Long-Range Transfer in DNA H: Schuster. G. B.. Ed: Springer: Heildel berg. 2004: p 103.

41. Wertz. T. E.: Bolton. J. R. Appendix D Experimental Methods; Spectronteter Perfomance in Electron Spin Resonance: Elementary Theory and Practical Applications, McGraw-Hill Book Company: New York. 1972, p 450.

42. Kittel, C. In Introduction to Solid State Plysics, Gth ed.; John Wiley: New York. 1991: $\mathrm{p} 413$.

43. Warman. J. M.: Haas. M. P. d.: Rupprecht. A. Chem. Phus Lett. 1996. 279.319 .

4. Briman, M.; Armitage. N. P.: Helgren, E.: Gruner. G. Nano Lett. 2004. 4.733 .

45. Nechtschein, M. Electon Spin Dramics in Handbook of Conducting Polmmers. 2nd ed.: Shotheim. T.. Elsenbaumer. R. L.. Reynolds. T. R.. Eds.: Marcel Dekker. Inc: New York. 1998: p 141 .

46. Endres, R. G. Cox, D. L.; Singh, R. P. P. Rev Moden Phys. 2004 76. 195 .

47. Kelley, S. O.: Barton, J. K. Science 1999. $283,375$.

48. Nunez. M. E.: Holmquist. G. P.: Barton. I. K. Biochemismy 2001. 40. 12465

49. Boon1. E. M.: Barton1. J. K. Cmm Opin. Siruct. Biol. 2002. 12.320.

50. Delaney, S.: Barton, J. K. J. Org. Chem. 2003, 68, 6475 .

51. Giese, B. Cwr Opin. Chem. Biol 2002. 6.612.

52. Okamoto, A.: Tanaka. K.: Saito, I. J. Am. Chem Soc. 2003, 125. 5066.

53. Henderson. P. T.: Tones. D.: Hampikian. G.: Kan. Y. Z.: Schuster. G. B. Proc. Natl Acad Sci. L.S.4. 1999. 96.8353.

54. Barnett, R. N.: Cleveland. C. L.: Joy. A.: Landman. U.: Schuster. G. B. Science 2001, 294. 567.

55. Lewis. F. D.; Liu. X. Y.: Liu, J. Q: Miller. S. E.; Haves, R. T: Wasielewski. M. R. Natme 2000. +06.51 .

56. Lewis. F. D.: Wu. Y. S.: Zhang. L. G: Zuo. X. B.: Hayes. R. I: Wasielewski. M. R. J. Am. Chent Soc. 2004. 126.8206

57. Mizoguchi, K.: Tanaka, S.; Ojima. M.: Sano. S.: Nagatori. M. Sakamoto, H.: Yonezawa, Y; Aok, Y: Sato. H.: Furukawa. K. Nakamura. T. J. Plns. Soc. Jph 2007, 76, 043801

58. Kaht1. O. Molectilar Hagnetisnt: VCH: New York. 1993.

59. Kittel. C. In Introduction to Solid State Phnsics. 6th ed.: Iohn Wiley: New York. 1991: p 423

60. Levy, L. P.; Reich. D. H.: Pteiffer. L.: West. K. Phsica B 1993. 189. 204 .

61. Kasumov, A. Y.: Kociak. M.: Gueron, S:- Reulet, B:- Volkov, V T.: Klinov. D. V.: Bouchiat. H. Science 2001. 291. 280.

62. Tagami. K.: Tsukada. M.: Wada. Y.: Iwasaki. T:: Nishide. H. J. Chent. Phs. 2003. 119.7491

63. Cai. Z. L.; Sevilla, M. D. J. Phus. Chem. B 2000. 104, 6942.

64. Minot. E. D: Yaish, Y.: Sazonova, V: McEuen, P. L. Nature 2004. 428.536.

65. Livolant. F.: Leforestier. A. Prog. Pohm. Sci. 1996. 21. 1115

66. Snalyukh. I. I.: Zribi. O. V.: Butler. J. C.: Laventowich. O. D.: Wong. G. C. L. Phws. Rew Lett. 2006. 96.177801.

67. Lorman, V; Podgornik. R.: Zeks. B. Phus. Rev Lett 2001, 87. 218101

68. Manna. F; Lorman, V; Podgornik. R; Zeks, B. Phys. Ren E 2007. 75. 03090I(R).

69. Komysher. A. A.: Lee. D. J.: Leikin1. S.: Wytnveen. A.: Zimmeman. 
S. B. Phus. Rev: Lett. 2005. 95. 148102.

70. Mesquita. M. V: Vasconcellos. A. R.: Luzzi. R.: Mascarenhas. S. Int. J. Quantum. Chem. 2005. 102. 1116.

71. Sension. R. J. Hatme 2007. H-16.740.

72. Lax, B.: Mavroides. J. G. Cyclohon Resonance in Solid State Plysics: Seitz. F., Tunbull. D.. Eds.: Academic Press: 1960: Vol. 11. 261 .

73. Bagguley. D. M. S.: Stradling. R. A.: Whiting. J. S. S. Proc. R. Soc. Lond 4 1961. 262.340

74. Bagguley. D. M. S.: Stradling. R. A.: Whiting. J. S. S. Proc. R.
Soc. Lond A 1961. 262.365.

75. Canali. C.: Jacoboni. C.: Nava. F.: Ottaviani. G.: Alberigi-Quaranta. A. Phos Rev: $B$ 1975. 12.2265.

76. Jacoboni, C. Nava. F; Canali. C.: Ottaviani. G. Phys. Res B 1981. 24. 1014 .

77. Santos, H. D. L.: Gray. J. L. IEEE Trans. Electron Davices 1988. 35. 1972.

78. Griftiths. D. T. Faraday's Law in Introduction to Electrodnnamics. 3rd ed: Prentice Hall: New Tersey. 1999: p 301. 